\title{
The measurement of the modal strain fields using digital shearography
}

\author{
H. Lopes ${ }^{1,1}$, J. Ribeiro ${ }^{1}$, M. Vaz ${ }^{2}$ and J. S. Gomes ${ }^{2}$ \\ ${ }^{1}$ ESTIG - Polytechnique Institute of Bragança -Cp. St Apolónia, Apr. 134 - 5301-857 Bragança \\ ${ }^{2}$ DEMEGI - Faculty of Engineering, University of Porto, R Dr Roberto Frias, 4200-465 Porto, Portugal
}

\begin{abstract}
This work presents a Michelson shearography interferometer configuration associated with stroboscopic double illumination technique for the measurement of modal rotation fields and their strain fields on a clamped circular aluminium plate. The speckle pattern is frozen by the synchronization between the LASER illumination and the modal vibration of the object. The quantitative evaluation is performed for each digital shearogram using a time modulation technique. The setup of double illumination LASER with out-of-plane opposite sensitivity allows the two phase maps measurement of the modal spatial gradient. The modal rotation and strain fields are extracted by the combination of this two digital phase maps. Image processing techniques are applied on the phase maps to obtain full-field measurements using a dedicated post-processing algorithm. Finally, is presented a comparison between the experimental measurement and the numerical solution.
\end{abstract}

\section{Introduction}

The strain field measurement in structures is a classical problem of the experimental mechanics. The strain fields allow the direct stress evaluation in structures. Hence, the stresses concentration can be direct detected and the mechanical components failure can be prevented. This allows the development of new powerful tools dedicated to the structural health monitoring.

The Michelson shearography interferometer is a full-field technique for measurement of slope or spatial displacement gradient [1-4]. The stroboscopic illumination is introduced by an acousto-optic modulator for the modal slope measurement on a clamped circular plate. The proposed method combines the information from two slopes with opposite sensitivity vectors, $\Delta_{+\theta}$ and $\Delta_{\theta}$, to obtain in-plane modal strain fields $\Delta_{I}[4]$. The sensitivity vector is adjusted during the measurement using two different illumination angles, see Fig. 1.

The phase maps of modal strain are obtained by the two slopes subtraction, according to the equation [4]:

$$
\Delta_{I}=\Delta_{+\theta}-\Delta_{-\theta}=\frac{4 \pi \delta \sin \theta}{\lambda} \frac{\partial u}{\partial x}
$$

\footnotetext{
1 e-mail : hlopes@ipb.pt
} 


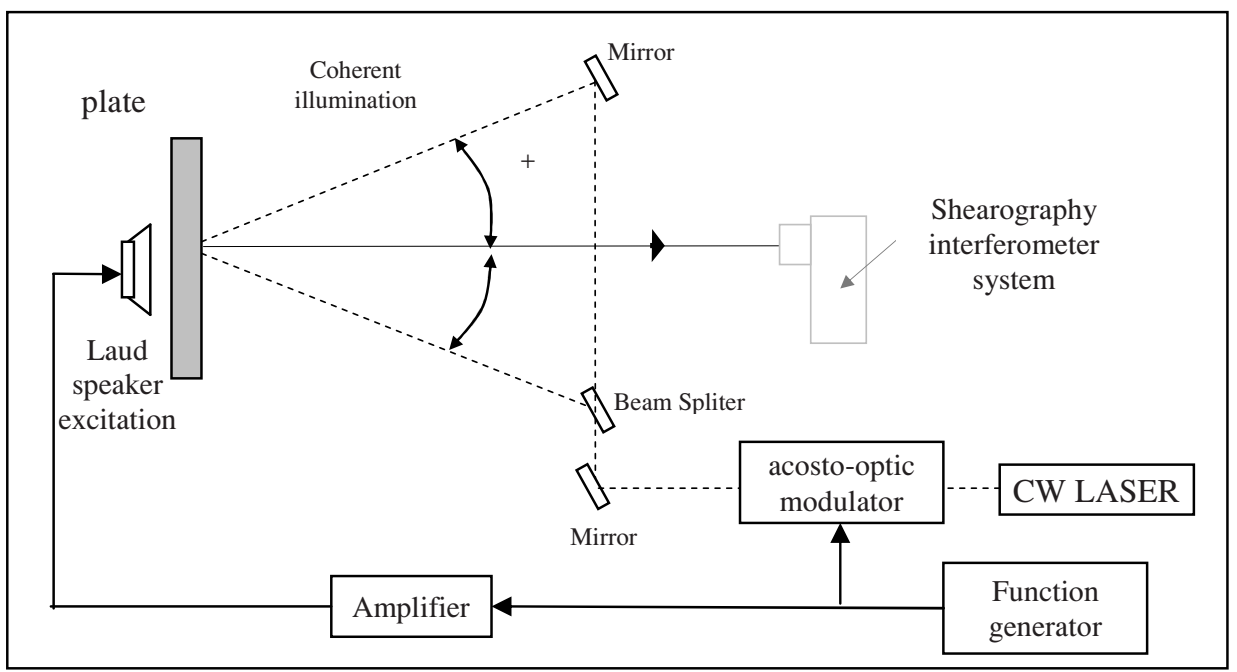

Fig. 1. Schematic diagram of experimental set-up.

\section{Conclusions}

The proposed methodology showed to be effective in modal slope and strain fields measurements. The numerical combination of the slope phase maps leads to the propagation of speckle noise and the destruction of the phase map information. Although, this noise can be eliminated using robust image processing techniques. Finally, was achieved a good agreement between the experimental and numerical results.

\section{References}

1. Araújo dos Santos JV, Lopes HMR., Vaz M, Mota Soares CM, Mota Soares CA, Freitas MJM de. Damage localization in laminated composite plates using mode shapes measured by pulsed TV holography. Composite Structures, 76(3):272-281, 2006

2. Hung MYY, Taylor CE. Speckle shearing interferometric camera - A tool for measurement of derivatives of surface displacements. Proceedings SPIE, 169-175, (1974).

3. Kreis T. Handbook of holographic interferometry: optical and digital methods. Weinheim: Wiley$\mathrm{VCH},(2005)$.

4. Wolfgang Seinchen, Lianxiang Yang, Digital Shearography -Theory and Application of Digital Speckle Pattern Shearing Interferometry, SPIE PRESS, (2003). 